\begin{tabular}{|l|l|l|l|l|}
\hline Cuadernos de Investigación Geográfica & 2000 & No 26 & pp. 131-145 & ISSN 0211-6820 \\
\hline
\end{tabular}

(c) Universidad de La Rioja

\title{
EL RÉGIMEN DEL EMBALSE DE YESA (CUENCA ALTA DEL RÍO ARAGÓN, PIRINEO CENTRAL) Y SU ADAPTACIÓN A LA VARIABILIDAD DEL RÉGIMEN FLUVIAL
}

\section{J. I. LÓPEZ MORENO \\ S. BEGUERÍA \\ J. M. GARCÍA RUIZ}

Instituto Pirenaico de Ecología, CSIC, Campus de Aula Dei, Apdo. 202, 50080-Zaragoza

C. electrónico: sbeguería@ipe.csic.es

\begin{abstract}
RESUMEN: Se estudia el régimen del embalse de Yesa, en la cuenca alta del río Aragón. El embalse se destina predominantemente a abastecer las áreas de regadio de las Bardenas, entre Navarra y Zaragoza, por lo que la gestión del embalse tiende a conseguir el máximo volumen de agua almacenada durante el mes de mayo, para liberar caudales durante todo el verano. El embalsado se produce desde el mes de octubre, aprovechando sobre todo las aguas altas de otoño y primavera. Se ban identificado tres patrones de gestión del embalse. Tales patrones no se distribuyen al azar en el tiempo, sino que han predominado en diferentes periodos desde la construcción del embalse, reflejando la existencia de cambios en el régimen fluvial.

ABSTRACT: The management regime (inflow and outflow) of the Yesa Reservoir, Upper Aragón River, is studied. The reservoir is mainly devoted to supply water for irrigation in Bardenas, between Navarra and Aragón. For this reason, the reservoir management tends to reach the maximum volume of water in May, in order to release relatively high flows in summer. The filling occurs since October, especially with the high autumn and spring discharges. Three management patterns bave been identified. Such patterns are not randomly distributed in time, but they have prevailed in different periods since the construction of the reservoir, showing the occurrence of changes in the fluvial regime.
\end{abstract}

Palabras clave: Embalse, Gestión de recursos hídricos, Régimen fluvial, Pirineo Central español.

Key words: Reservoir, Water resources management, Fluvial regime, Central Spanish Pyrenees.

\section{Introducción}

Es bien conocida la importancia de los embalses en la articulación del territorio por la complejidad de las funciones que desarrollan (almacenamiento de agua para riego y suministro urbano e industrial, producción hidroeléctrica, control de avenidas y uso recreativo). Esa es la razón por la que el número de grandes embalses ha pasado de 500 a más de 42.000 en el mundo a lo largo del siglo XX (Morris 
et al., 1997), entendiendo por gran embalse, aquel cuya presa supera los 15 metros de altura.

Sin embargo, los estudios sobre diferentes aspectos hidrológicos y geomorfológicos de los embalses no han experimentado un desarrollo acorde con su incremento en número e importancia, estando principalmente orientados a investigar los cambios hidrológicos que se producen aguas abajo de la presa (Higgs \& Petts,1988; Zsuffa, 1999; Picher, 1990; Jiongxin, 1990), las variaciones geomorfológicas en el cauce afectado (Anders, 2000; Al-Taiee, 2000), diversos aspectos ecológicos (Thomas, 1996; Giers et al., 1998) y de una forma bastante extensa la pérdida de capacidad de almacenamiento por procesos de aterramiento (Morris et al., 1997; Petkovic et al., 1999; Harden, 1993; Summer, 1990; Verstraeten \& Poesen, 2000; Tamburino et al., 1990). Algunos trabajos se centran en la importancia de los usos del suelo y en las características físicas de la cuenca drenada para explicar el ritmo de sedimentación en embalses (Harden, 1993; Sherwood et al., 1993; McIntyre, 1993; Valero-Garcés et al., 1998).

Es evidente que la construcción de embalses representa importantes modificaciones hidrológicas y geomorfológicas, tanto mayores cuanto mayor es la capacidad de almacenamiento del embalse en relación con la aportación anual. Desde un punto de vista hidrológico tales modificaciones están relacionadas tanto con las funciones para las que ha sido construido el embalse como con las características del régimen fluvial. En el área mediterránea los grandes embalses tienen como función principal el abastecimiento de agua para el regadío, especialmente durante la estación cálida y seca. Esto implica una alteración profunda del régimen fluvial, que en general, presenta los caudales más elevados a lo largo de la estación fría. El régimen de embalsado debería tender a alcanzar el máximo volumen almacenado justo al inicio de la estación cálida, durante la cual tendría lugar el proceso de desembalsado. La peculiaridad de las precipitaciones en el área mediterránea, con una elevada concentración del caudal en unos pocos eventos, explica un aprovechamiento intensivo de los periodos de avenida para aumentar la reserva de agua en los embalses, contribuyendo así a la reducir la intensidad de las crecidas aguas abajo (laminación de crecidas). En todo caso se trata de un fenómeno complejo en el que interfieren régimen fluvial y demanda.

En este trabajo se analiza el ritmo de llenado y vaciado del mayor embalse de los afluentes pirenaicos del Ebro, el embalse de Yesa, ubicado en la cuenca alta del río Aragón. El principal objetivo es estudiar la gestión del embalse en función de las variaciones interanuales experimentadas por el régimen fluvial. Se trata de un embalse destinado principalmente a abastecer las áreas de regadío de las Bardenas (60.701 ha entre Navarra y Aragón) por medio del canal del mismo nombre.

\section{El área de estudio}

El embalse de Yesa drena una superficie de $2181 \mathrm{Km}^{2}$ en la cuenca superior del río Aragón (Fig. 1). Por la derecha recibe a sus afluentes más importantes procedentes de las sierras más elevadas del Alto Aragón Occidental, destacando los ríos 
Estarrún, Aragón Subordán, Veral y Esca. Por la izquierda, en cambio, la superficie drenada es muy pequeña y los afluentes son de escasa importancia.

El relieve se ordena en una serie de bandas con orientación general oeste-este, distinguiéndose de norte a sur los siguientes sectores:

- Pirineo Axial, constituido por materiales paleozoicos que presentan una gran variedad litológica: pitones volcánicos (Anayet, $2559 \mathrm{~m}$ ), calizas, pizarras y arcillas, lo que se refleja en un paisaje muy heterogéneo, con áreas de topografia relativamente suave y áreas escarpadas.

- Las Sierras Interiores, constituidas por calizas y areniscas mesozoicas. El relieve es muy accidentado, con grandes escarpes verticales. En este sector se alcanzan las mayores altitudes del área de estudio (Collarada, 2886 m, Aspe, 2645 m, Bisaurín, $2668 \mathrm{~m}$, Peña Forca, $2390 \mathrm{~m}$ ).

- Al sur de las Sierras Interiores, el sector del flysch eoceno ocupa un amplio territorio. El relieve se caracteriza por suaves divisorias, cuyas cotas máximas apenas superan los $2000 \mathrm{~m}$.

- La Depresión Interior, donde se ubica el embalse de Yesa, se modela en margas eocenas, dando lugar a un amplio corredor cuyo fondo oscila entre los 600 y 850 $\mathrm{m}$, dominado por la presencia de glacis y terrazas.

- El área meridional está ocupada por conglomerados, areniscas y arcillas continentales. Corresponde al Prepirineo, con relieves suavemente plegados.

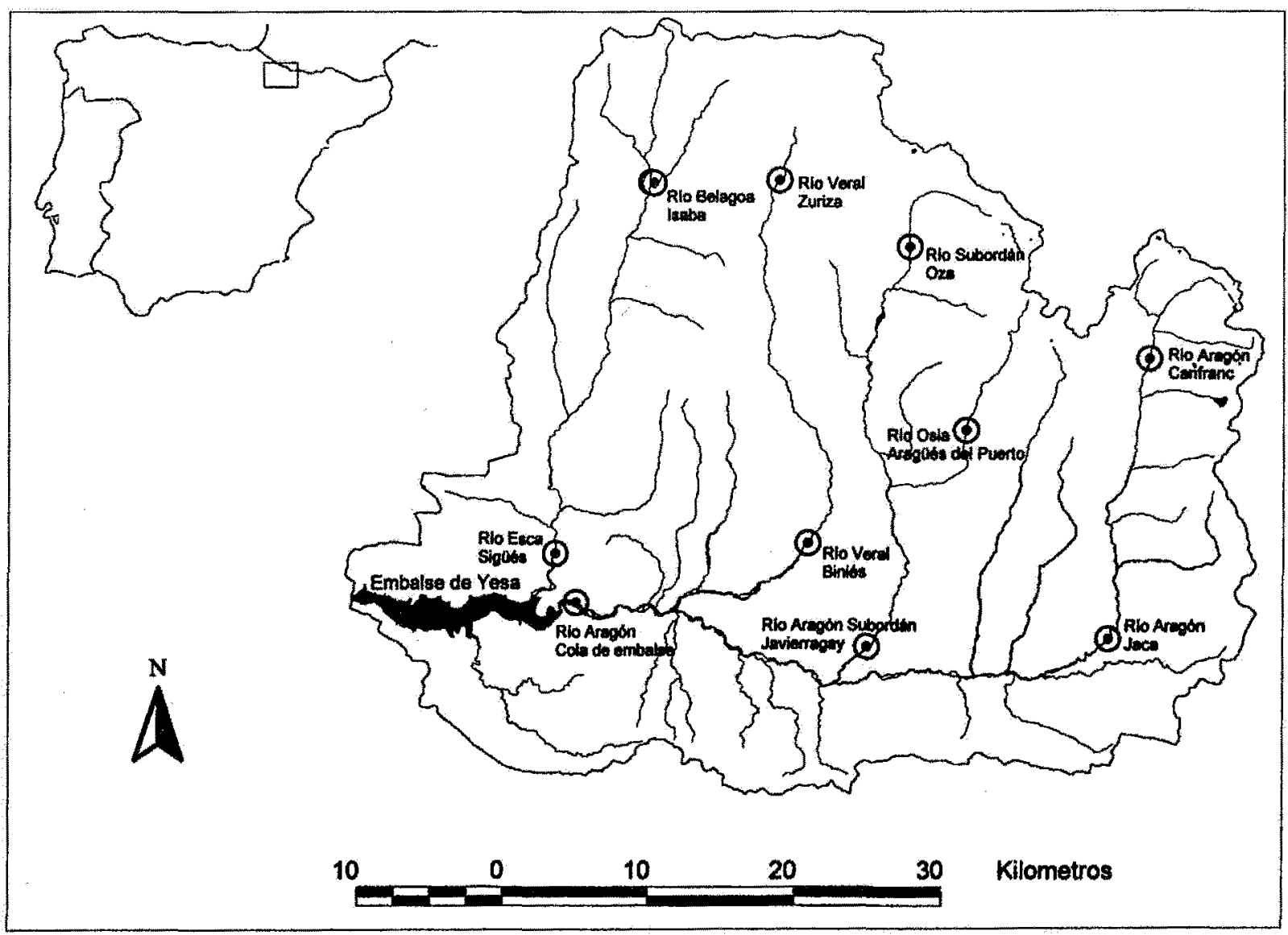

Figura. 1. La cuenca superior del río Aragón, con indicación de la localización de las estaciones de aforo. 
Precipitaciones y temperaturas muestran un claro gradiente hacia el sur y hacia el este. Debe tenerse en cuenta que la cuenca del río Aragón ocupa un espacio de transición entre las influencias atlánticas (al norte y oeste) y las influencias mediterráneas (al sur y este). La consecuencia es que las precipitaciones más elevadas se registran en el extremo septentrional de la cuenca (más de $1500 \mathrm{~mm}$ ). Al sur de las Sierras Interiores las precipitaciones descienden rápidamente hasta $820 \mathrm{~mm}$ en Jaca y $730 \mathrm{~mm}$ en Artieda. La Depresión Interior muestra claros rasgos de influencia mediterránea.

En cuanto a las temperaturas, el gradiente general refleja variaciones relacionadas con el relieve y la posición latitudinal, de manera que la temperatura disminuye de sur a norte. Al penetrar en condiciones montanas, los valores descienden rápidamente hasta alcanzar medias anuales muy bajas (en torno a $8^{\circ} \mathrm{C}$ en Canfranc). Por encima de los $1200 \mathrm{~m}$ hiela más de 130 días al año entre octubre y mayo (Creus, 1983). En la cuenca del tío Aragón se ha estimado que durante la estación fría (de noviembre a abril) la isoterma de $0^{\circ} \mathrm{C}$ se localiza a $1549 \mathrm{~m}$ (García Ruiz et al., 1986) lo que da idea de la importancia de la innivación.

La vegetación ha sido profundamente alterada por las actividades humanas. La agricultura cerealista ha dominado al sur de las Sierras Interiores, especialmente en los depósitos cuaternarios de la Depresión Interior y en las laderas solanas y fondos de valle del sector del flysch. Los cambios de uso del suelo durante el siglo $\mathrm{XX}$ han conducido a un rápido proceso de abandono de los campos localizados en laderas, afectados actualmente por una recolonización por el matorral. Algunas de las laderas cultivadas han sido reforestadas y, en gran parte de los casos, los campos de cereal han sido sustituidos por prados (García Ruiz \& Lasanta, 1993). Los bosques naturales forman manchas extensas y discontinuas por encima de $1400 \mathrm{~m}$ s.n.m., siendo muy irregular su límite superior debido a la deforestación. A partir de $1800 \mathrm{~m}$ el piso subalpino forma extensos pastos de aprovechamiento estival, alternando con pequeños bosques de pino negro. El piso alpino se inicia aproximadamente a $2300 \mathrm{~m}$.

\section{Métodos}

Se ha utilizado la información facilitada por la Confederación Hidrográfica del Ebro, en este caso datos diarios de las estaciones de aforo instaladas en el río Aragón y en sus afluentes más importantes (Fig, 1).

A partir de los datos de caudal diario se han obtenido los caudales mensuales y anuales $\left(\mathrm{m}^{3} / \mathrm{s}\right)$, que se han convertido tambien en datos de aportación mensual y anual $\left(\mathrm{Hm}^{3}\right)$. Los datos disponibles llegan hasta el año hidrológico 1995/96. La Tabla 1 informa sobre la longitud de las diferentes series hidrológicas utilizadas.

Dados los objetivos de este trabajo, se ha prestado especial atención a los datos de entrada y salida del embalse. Para evaluar la entrada total al embalse se han sumado los datos registrados en el río Aragón, a cola de embalse, y los medidos en el río Esca a su paso por Sigüés, poco antes de incorporarse al río Aragón en el embalse de Yesa. El resto de las entradas corresponde a barrancos o ríos de pequeña entidad que se encuentran sin aforar y las precipitaciones directas sobre el embalse, que 
representan un porcentaje muy bajo de las aportaciones. Para estimar las salidas se han sumado los datos registrados en la estación que se sitúa en el pie de la presa y los que se derivan al Canal de Bardenas. El resto de salidas son pérdidas por el subsuelo, muy poco importantes en el caso del embalse de Yesa, y pérdidas por evaporación.

Tabla 1. Longitud de las diferentes series bidrológicas utilizadas.

\begin{tabular}{|l|l|l|}
\hline Estación de aforos & Río & Años \\
\hline Canfranc & Aragón & $1971-96$ \\
Jaca & Aragón & $1931-98$ \\
Aragüés del Puerto & Osía & $1993-98$ \\
Oza & Subordán & $1992-95$ \\
Javierregay & Aragón Subordán & $1952-97$ \\
Zuriza & Veral & $1951-95$ \\
Biniés & Veral & $1949-96$ \\
Isaba & Belagoa & $1951-68$ \\
Isaba & Esca & $1993-95$ \\
Sigüés & Esca & $1951-95$ \\
Yesa-Cola embalse & Aragón & $1959-95$ \\
Yesa-Pie de presa & Aragón & $1913-99$ \\
\hline
\end{tabular}

De los datos de aportación que influyen directamente sobre el embalse se ha obtenido, mediante regresión lineal, la tendencia existente desde la construcción del embalse, por su posible influencia en los patrones de embalsado y desembalsado.

Por último, se han analizado los datos del volumen diario acumulado en el embalse de Yesa. Para identificar distintos patrones de gestión del embalse se ha elaborado una base de datos con información mensual de aportaciones al embalse, salidas y volumen almacenado, en $\mathrm{Hm}^{3}$. Los datos han sido normalizados y se les ha aplicado un análisis cluster (clasificación jerárquica) según el método de Ward. Los análisis se han realizado con el paquete StatGraphics 2.1.

\section{Resultados}

\subsection{El régimen del río Aragón en Yesa}

Los dos principales ríos que drenan al embalse de Yesa son el Aragón y el Esca con regímenes bien diferenciados.

El río Aragón aporta una media de $1019 \mathrm{Hm}^{3}\left(32.6 \mathrm{~m}^{3} / \mathrm{s}\right)$ anuales al embalse de Yesa. La Fig. 2 muestra la distribución mensual del caudal, con aguas relativamente altas durante toda la estación fría e incremento de los caudales medios en primavera, con máximo en mayo $\left(58.3 \mathrm{~m}^{3} / \mathrm{s}\right)$. A partir de junio se inicia un fuerte des- 
censo hasta los niveles más bajos del verano. Este régimen es el resultado de la diversidad de influencias y de regímenes fluviales dominantes en los diferentes sectores de la cuenca. Así en la cabecera del río Aragón Subordán (estación de aforo de Oza), la importancia de los procesos de retención y fusión nival se manifiestan en aguas bajas invernales y un pico muy fuerte de aguas altas en abril y mayo, debido a la fusión nival y a un recrudecimiento de las precipitaciones (García Ruiz et al., 1985). A medida que los ríos drenan territorios más bajos altitudinalmente, la influencia pluvial es mayor y tiende a amortiguar los fuertes contrastes estacionales generados por la influencia nival. Ese es el caso del río Aragón Subordán en Javierragay, del río Veral en Biniés y del mismo río Aragón a la entrada del embalse de Yesa (Fig. 2).

En cambio, el río Esca en Sigüés muestra un régimen claramente oceánico con aguas altas entre diciembre y abril (máximo en febrero), coincidiendo con la mayor actividad de los frentes procedentes del Atlántico (Fig.2). Desde abril el caudal desciende rápidamente hasta el verano. La aportación media anual de este río al embalse de Yesa es de $353 \mathrm{Hm}^{3}\left(12.1 \mathrm{~m}^{3} / \mathrm{s}\right)$.

Rio Aragón en Cantrane

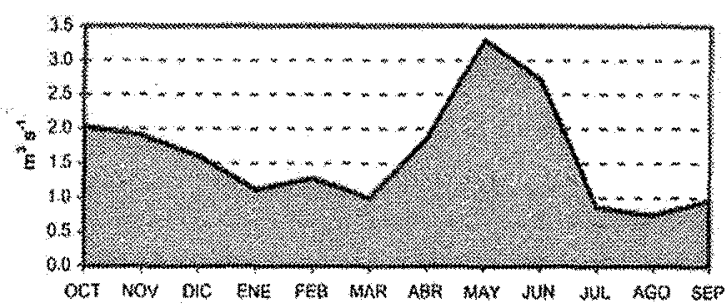

Rio Voral en Binís

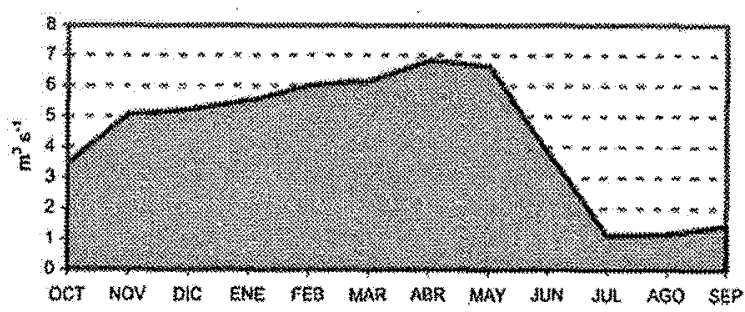

Río Aragón en la cola de embalse de Yosa (Yesa C.E.)

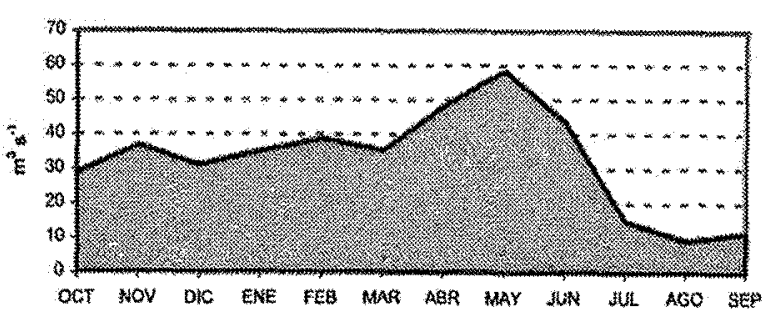

Rio Aragón Subordản en Javierragay

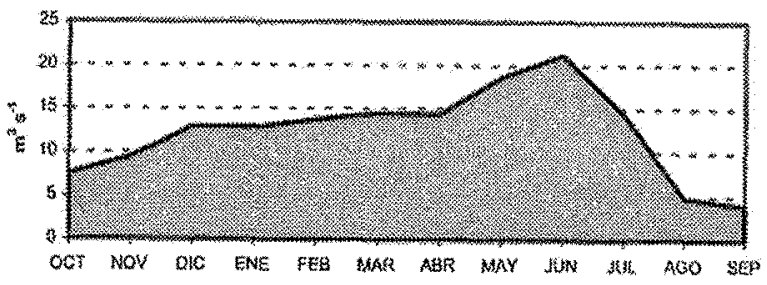

Rio Esca en Sigues

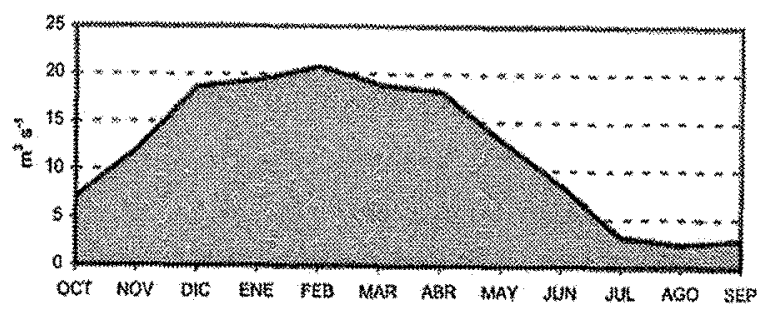

Entradas al embalse de Yesa (Yesa C.E. * hio Esca en Sigdès)

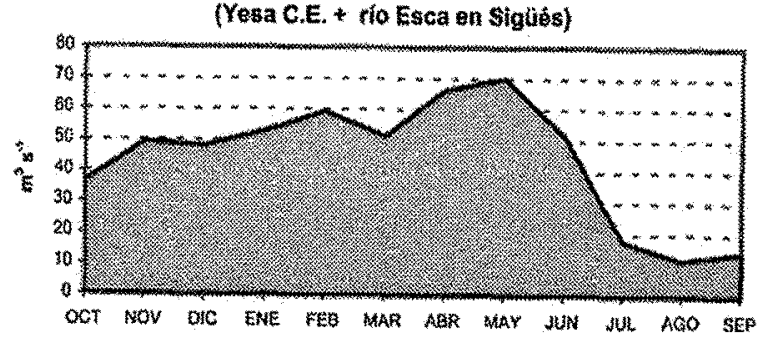

Figura. 2. Régimen fluvial en diversas estaciones de aforo de la cuenca superior del río Aragón. 
La suma de los ríos Aragón y Esca representa una aportación total anual de 1372 $\mathrm{Hm}^{3}\left(44 \mathrm{~m}^{3} / \mathrm{s}\right)$. La curva de distribución mensual de los caudales refleja especialmente el régimen del río Aragón, debido a su mayor importancia. De ahí que el máximo caudal medio se alcance en el mes de mayo. De todas formas, la influencia del río Esca se deja sentir en la mayor relevancia relativa de los caudales invernales y en la agudización del pico secundario de febrero (Fig. 2).

Tabla 2. Número medio de crecidas mensuales y anuales en los ríos Aragón y Esca.

\begin{tabular}{|lcc|}
\hline & $\begin{array}{c}\text { Río Esca, } \\
\text { Sigüés }\end{array}$ & $\begin{array}{c}\text { Río Aragón, } \\
\text { cola de embalse }\end{array}$ \\
\hline Octubre & 0,28 & 0,42 \\
Noviembre & 0,68 & 0,58 \\
Diciembre & 1,14 & 0,33 \\
Enero & 1,19 & 0,39 \\
Febrero & 1,10 & 0,36 \\
Marzo & 0,81 & 0,25 \\
Abril & 0,75 & 0,42 \\
Mayo & 0,48 & 0,53 \\
Junio & 0,17 & 0,31 \\
Julio & 0,00 & 0,06 \\
Agosto & 0,02 & 0,03 \\
Septiembre & 0,02 & 0,00 \\
\hline Anual & 6,63 & 3,59 \\
\hline
\end{tabular}

Para el estudio de las crecidas se han considerado aquellos días cuyo caudal iguala o supera en cinco veces el caudal medio anual (ver Tabla 2), es decir, $163 \mathrm{~m}^{3} / \mathrm{s}$ en el río Aragón en la cola del embalse, $60.5 \mathrm{~m}^{3} / \mathrm{s}$ en el río Esca y $220 \mathrm{~m}^{3} / \mathrm{s}$ en el caso de las entradas totales al embalse (suma de los ríos Aragón y Esca). Si se tienen en cuenta estas útimas, el periodo de crecidas se reparte a lo largo de toda la estación fría, con máximos en otoño y principios de invierno, siendo nula su ocurrencia en verano. Si se distingue entre los ríos Aragón y Esca se comprueba que en el primero la mayor frecuencia se da en otoño y en primavera, con un descenso en invierno, mientras que en el segundo el invierno acapara el mayor número de crecidas. La influencia de las tormentas estivales en la cuenca del río Aragón explica la existencia de una pequeña proporción de crecidas en verano. La crecida más elevada registrada en el río Aragón corresponde al 1 de junio de 1974 , con $824 \mathrm{~m}^{3} / \mathrm{s}$; en el río Esca corresponde al 17 de diciembre de 1962, con $256 \mathrm{~m}^{3} / \mathrm{s}$.

\subsection{El embalse de Yesa}

La presa del embalse de Yesa está situada en la provincia de Navarra, término municipal de Yesa, aunque la mayor parte del embalse inunda tierras de la provincia de Zaragoza. En el momento de su construcción (año 1959) tenía una capacidad de $470 \mathrm{Hm}^{3}$, si bien una batimetría realizada en 1986 estimó una capacidad de 450.3 $\mathrm{Hm}^{3}$, debido a procesos de aterramiento que representaron una aportación anual de sedimentos de $0.79 \mathrm{Hm}^{3}$. La altura de la presa es de $74 \mathrm{~m}$. 
El uso fundamental del embalse es el suministro de agua para regadío, mediante la derivación de agua al Canal de Bardenas, a la par que desempeña un importante papel en el control de crecidas.

En la Fig. 3 se refleja la evolución media del volumen de agua embalsada, así como las entradas (correspondientes a la suma de los caudales mensuales de los ríos Aragón y Esca) y las salidas (suma de caudales evacuados desde el embalse, es decir, por los aliviaderos y por el Canal de Bardenas). Cada columna de la Fig. 3 indica el estado del embalse al final del mes correspondiente. En cambio, las entradas y las salidas se refieren a medias mensuales obtenidas a partir de datos diarios. El agua almacenada en el embalse muestra un progresivo incremento desde el mes de septiembre hasta alcanzar el máximo durante el mes de mayo, con valores en torno a $400 \mathrm{Hm}^{3}$. Si se comparan las curvas de entradas y salidas se comprueba que las primeras son superiores a las segundas desde el mes de octubre hasta el mes de junio, lo que pertmite el llenado del embalse en otoño, invierno y primavera. Las salidas, en cambio, superan a las entradas durante los meses de verano, de forma todavía poco acusada en junio y de manera muy evidente en julio, agosto y septiembre. Es importante tener en cuenta que la mayor separación entre entradas y salidas a favor de las primeras se produce durante el otoño (noviembre) y la primavera (mayo), coincidiendo con dos periodos de aguas altas en el río Aragón. En pleno invierno las diferencias entre entradas y salidas se reducen al mínimo, sobre todo en febrero y marzo.

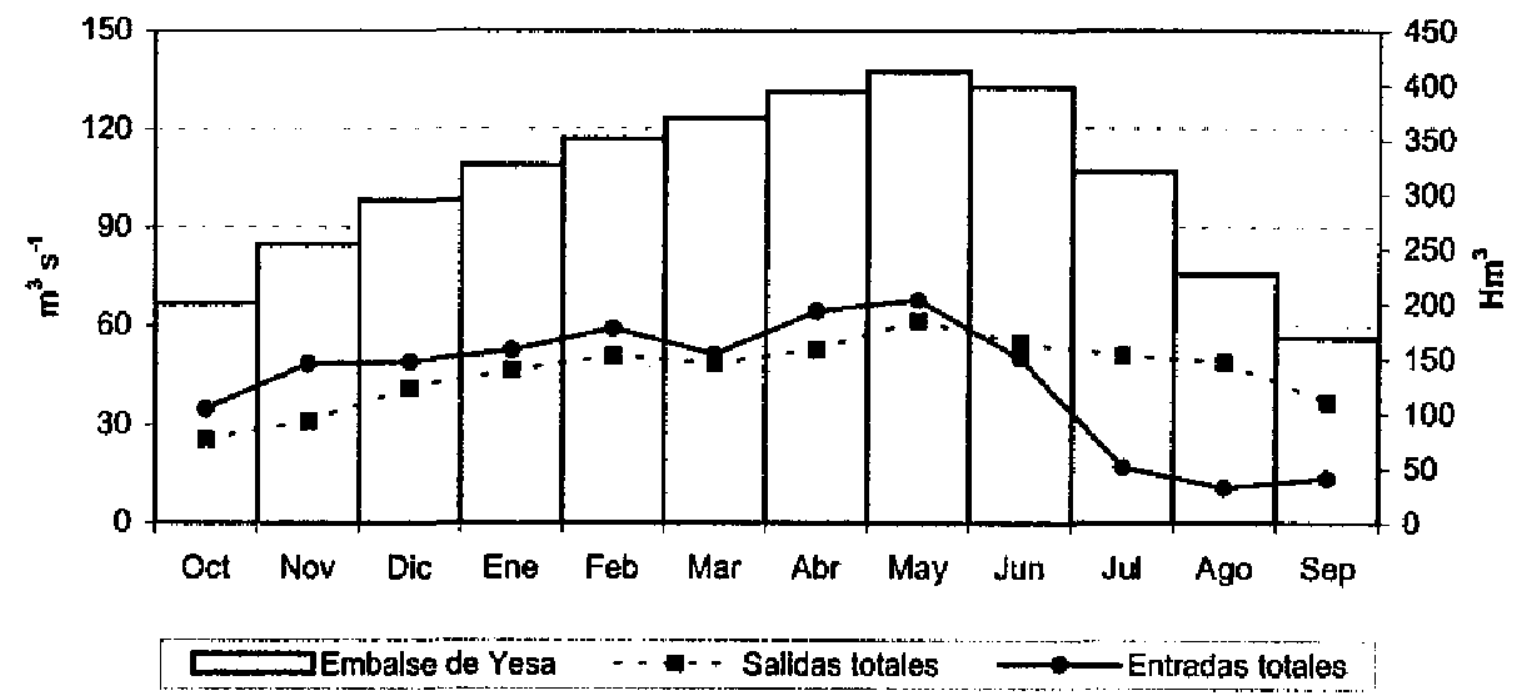

Figura. 3. Volumen mensual medio en el embalse de Yesa y balance de entradas y salidas

La Fig. 4 muestra la evolución de los caudales desembalsados a lo largo del año, distinguiendo entre el rio Aragón y el canal de Bardenas. No resulta sorprendente que sus curvas muestren tendencias opuestas: así, el río Aragón presenta, aguas abajo del embalse de Yesa, aguas altas desde noviembre a abril, con máximo en febrero, mientras que el Canal de Bardenas lleva caudales muy bajos desde octubre hasta abril, y caudales elevados muy superiores a los del mismo río Aragón de mayo a agosto. En cierto modo, el río Aragón reproduce aguas abajo del embalse un régimen oceánico similar al del zío Esca, lo que significa que el embalse de Yesa retiene especialmente los caudales de aguas altas del río Aragón (otoño y primavera). 


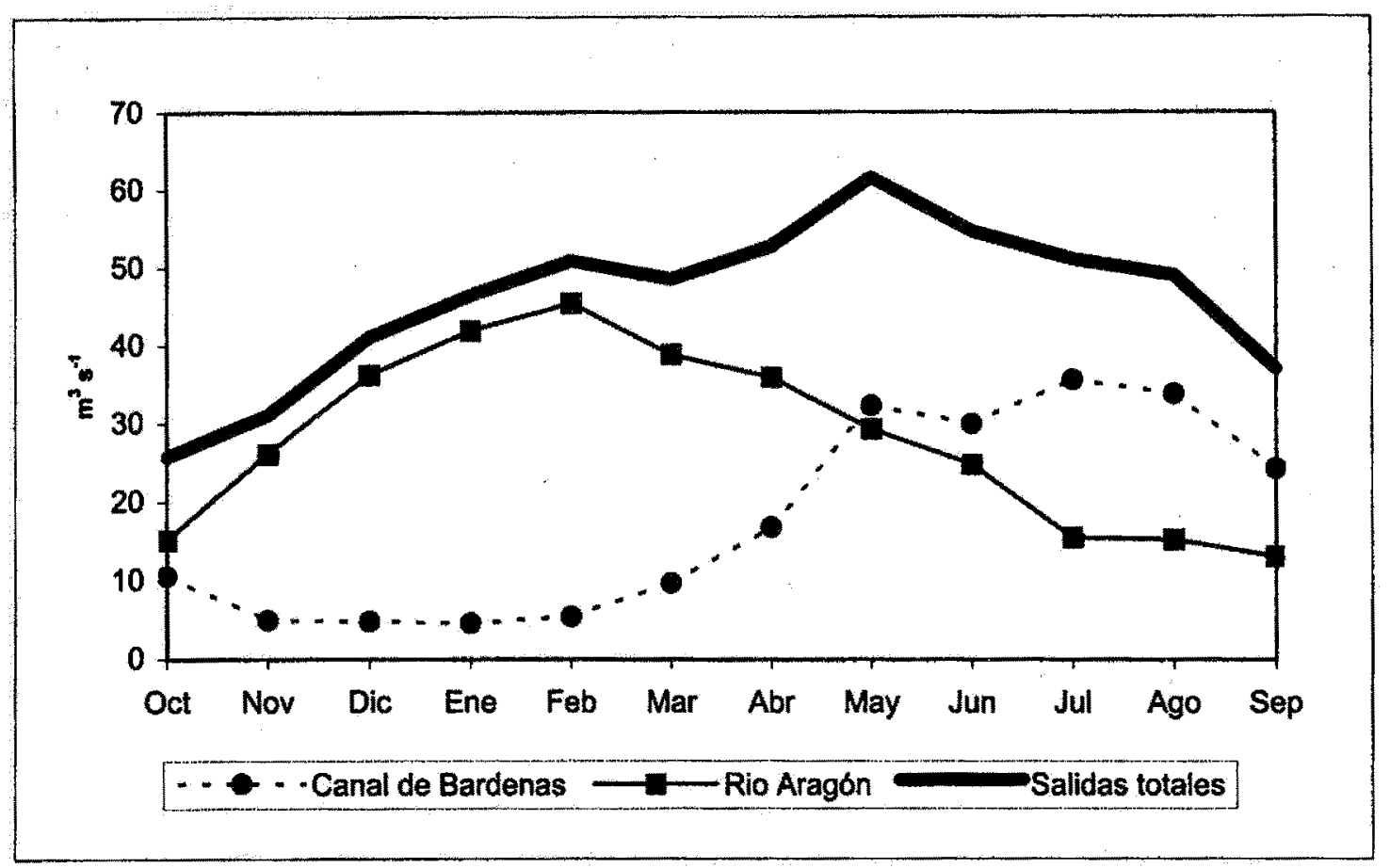

Figura. 4. Evolución mensual de los caudales desembalsados desde el embalse de Yesa.

Las crecidas de octubre y noviembre son en gran parte retenidas para ayudar a un rápido llenado inicial del embalse. En lo tres meses siguientes sigue efectuándose un laminado de avenidas, pero su frecuencia e intensidad es sensiblemente inferior a las otoñales. De ahí que se incremente en muy poco el volumen embalsado. Son las crecidas de primavera las que contribuyen al llenado definitivo del embalse. De todas formas, la gestión del embalse frente a las avenidas depende tanto de la intensidad como del estado en que se encuentra el embalse.

\subsection{Fluctuaciones del volumen embalsado}

La gestión del embalse está, pues, orientada a conseguir el máximo volumen almacenado en el mes de junio, para responder a una demanda elevada, precisamente durante el periodo en el que la aportación al embalse es muy baja.

Para lograr este objetivo, sus gestores deben adaptarse a las diferentes condiciones hidrológicas que se presentan cada año, teniendo en cuenta tanto el volumen total de agua que llega al embalse, como las aportaciones a lo largo del año. Es importante recordar que el régimen del río Aragón a la entrada del embalse de Yesa muestra a la vez influencias pluviales y nivales, de manera que existe una notable diversidad interanual en el reparto de los caudales. Así, las lluvias otoñales muestran una gran irregularidad, pudiendo retrasarse hasta cerca del invierno o, por el contrario, producir avenidas de gran envergadura; de igual forma, las avenidas primaverales dependen no solo de la intensidad y duración de las lluvias, sino también de la importancia de las precipitaciones invernales y del volumen de agua retenida en forma sólida. La gestión del embalse depende, obviamente, de esta variabilidad climática, pero siempre trata de asegurar un volumen de agua embalsada a finales de primavera, a la vez que se deja un margen de seguridad para laminar posibles crecidas. 
La identificación de diferentes patrones de gestión del embalse de Yesa se ha realizado mediante un análisis cluster, que ha permitido distinguir tres patrones de embalsado y desembalsado (Fig. 5). Los tres grupos se encuentran muy condicionados por el volumen almacenado al comienzo del año hidrológico, el ritmo de aportación de las entradas, entre los meses de octubre y junio, y las aportaciones totales del año hidrológico.

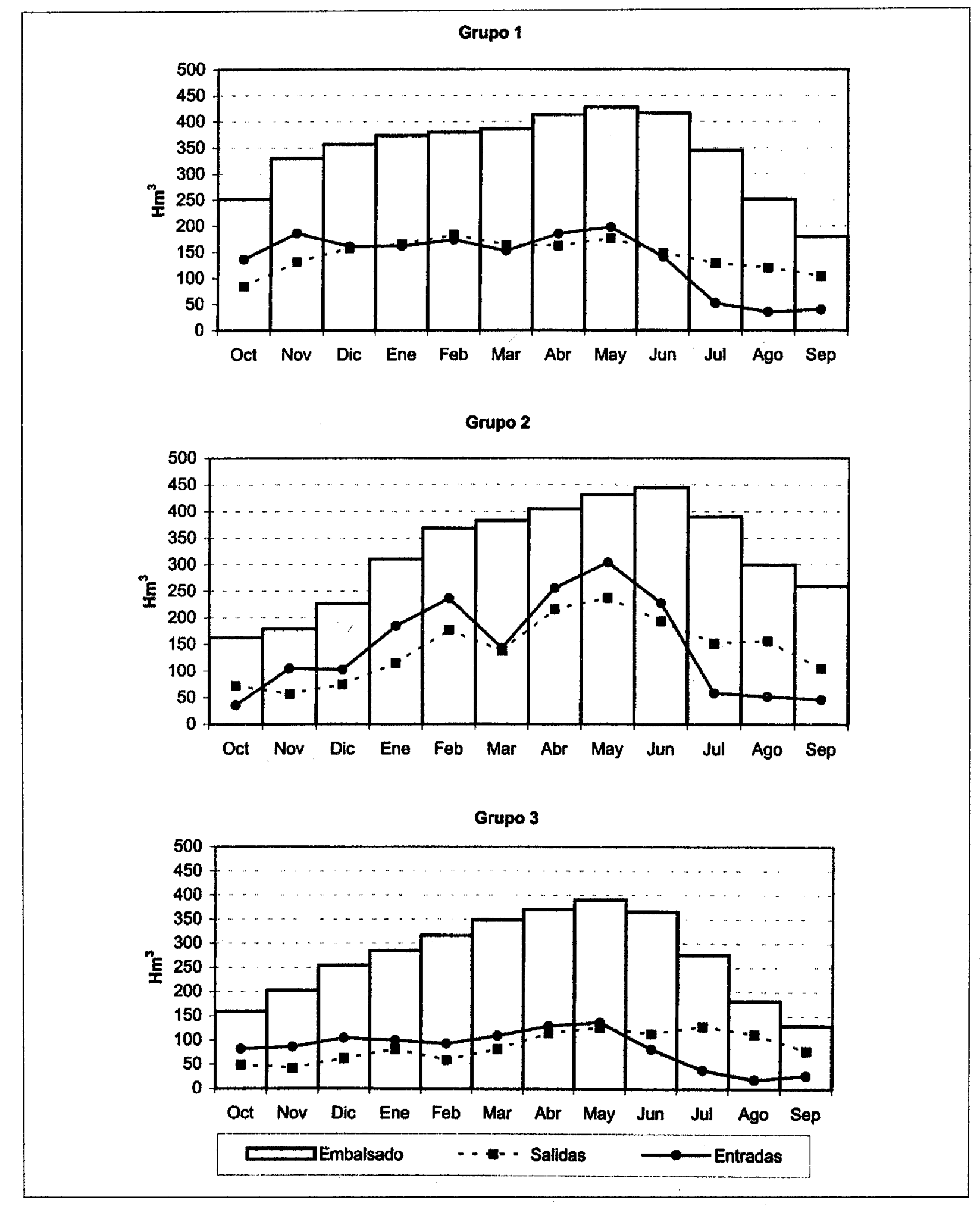

Figura. 5. Patrones de gestión del embalse de Yesa, a partir de los centroides de grupo obtenidos mediante análisis cluster. 
El grupo 1 parte de un elevado volumen de agua almacenada (en torno a 225 $\mathrm{Hm}^{3}$ ). Durante los meses de octubre a diciembre experimenta un rápido incremento, debido a las elevadas aportaciones de los ríos Aragón y Esca, muy superiores a las salidas. De enero a marzo el incremento de la reserva es muy suave o inexistente debido al equilibrio entre los valores de entrada y salida. En los siguientes meses (de abril a mayo) el volumen vuelve a aumentar hasta alcanzar en mayo la máxima cantidad almacenada (por término medio, alrededor de $425 \mathrm{Hm}^{3}$ ). Este grupo se basa en el incremento del volumen embalsado durante dos periodos (otoño y primavera) separados por un periodo estable en el que, aunque las aportaciones pueden llegar a ser elevadas (especialmente en el río Esca), se pretende establecer un margen de seguridad frente a posibles avenidas de estación fría. El ritmo de entradas al embalse reproduce con bastante fidelidad el régimen medio del río Aragón.

En éste, como en los demás grupos, los meses de julio a septiembre se caracterizan por un claro descenso del volumen embalsado, al ser las entradas de agua mucho menores que las salidas. En el grupo 1 el volumen almacenado al final del año hidrológico suele estar alrededor de $200 \mathrm{Hm}^{3}$.

El grupo 2 inicia el año hidrológico con un nivel de embalsado inferior a 200 $\mathrm{Hm}^{3}$. Durante los meses de octubre a diciembre los niveles de entrada son claramente inferiores a los del grupo 1, manteniendo niveles bajos de embalsado durante todo el otoño. El mayor aumento en el ritmo de almacenamiento de agua se produce a partir del mes de enero, cuando se separan de forma nítida entradas y salidas. Un descenso en las entradas en el mes de marzo da lugar a una estabilización del volumen embalsado, para incrementarse de nuevo en primavera y alcanzar el máximo en junio, con un volumen similar al del grupo 1 (en torno a $440 \mathrm{Hm}^{3}$ ). Es evidente que el grupo 2 está relacionado con una ausencia de precipitaciones importantes durante el otoño y que el ritmo de llenado se basa en las lluvias invernales y en las siempre seguras aguas altas primaverales.

El grupo 3 parte de un volumen inicial muy bajo (alrededor de $141 \mathrm{Hm}^{3}$ ) y se mantiene por debajo de los otros dos grupos durante todo el año hidrológico. El perfil de llenado y vaciado de este grupo muestra una tendencia suavemente creciente de octubre a mayo, hasta alcanzar un volumen máximo en junio en torno a $380 \mathrm{Hm}^{3}$, con un rápido descenso hasta volúmenes inferiores a $150 \mathrm{Hm}^{3}$ en septiembre. La curva correspondientes a las entradas refleja la ausencia de un periodo con grandes aportaciones, pudiendo apreciarse un ligero pico en mayo. Las aportaciones de otoño e invierno se mantienen a un nivel muy bajo.

La distribución de cada uno de los grupos durante el periodo de funcionamiento del embalse (Tabla 3) sugiere que existe una marcada tendencia temporal. Así, el Grupo 1 domina durante la primera década tras la construcción del èmbalse y disminuye progresivamente su ocurrencia hasta desaparecer en los años noventa. De igual forma, el Grupo 2 solo tiene presencia al principio del periodo de estudio, mientras que el Grupo 3 sigue una evolución contraria, con incremento hasta la década de los noventa, momento en que se hace exclusivo. Los grupos 1 y 3, cada uno representado con 16 años, son los dominantes, pero uno lo hace al principio del funcionamiento del embalse de Yesa y el otro al final, indicando que el régimen de llenado del embalse ha cambiado espectacularmente. 
Tabla 3. Distribución temporal de los diferentes patrones de gestión del embalse.

\begin{tabular}{|c|c|c|c|}
\cline { 2 - 4 } \multicolumn{1}{c|}{} & Grupo 1 & Grupo 2 & grupo 3 \\
\hline $1960-1969$ & 8 & 2 & 0 \\
$1970-1979$ & 5 & 3 & 2 \\
$1980-1989$ & 3 & 0 & 7 \\
$1990-1996$ & 0 & 0 & 7 \\
Total (1959-1996) & 16 & 5 & 16 \\
$\%$ & 43,2 & 13,5 & 43,3 \\
\hline
\end{tabular}

Esta evolución sólo puede explicarse por cambios en el régimen de aportaciones de los ríos Aragón y Esca. La Fig. 6 refleja la pendiente de las tendencias observadas a partir de regresiones lineales en el caudal de los ríos Esca y Aragón y en el Canal de Bardenas, para el periodo 1959-1996. Todos los casos analizados muestran una tendencia negativa, excepto el Canal de Bardenas que ha experimentado una mínima tendencia positiva, prueba de que la demanda se mantiene estable o se incrementa ligeramente. El río Esca presenta una pequeña tendencia negativa, mientras que el río Aragón ha reducido claramente las aportaciones, lo que redunda en unas menores entradas al embalse.

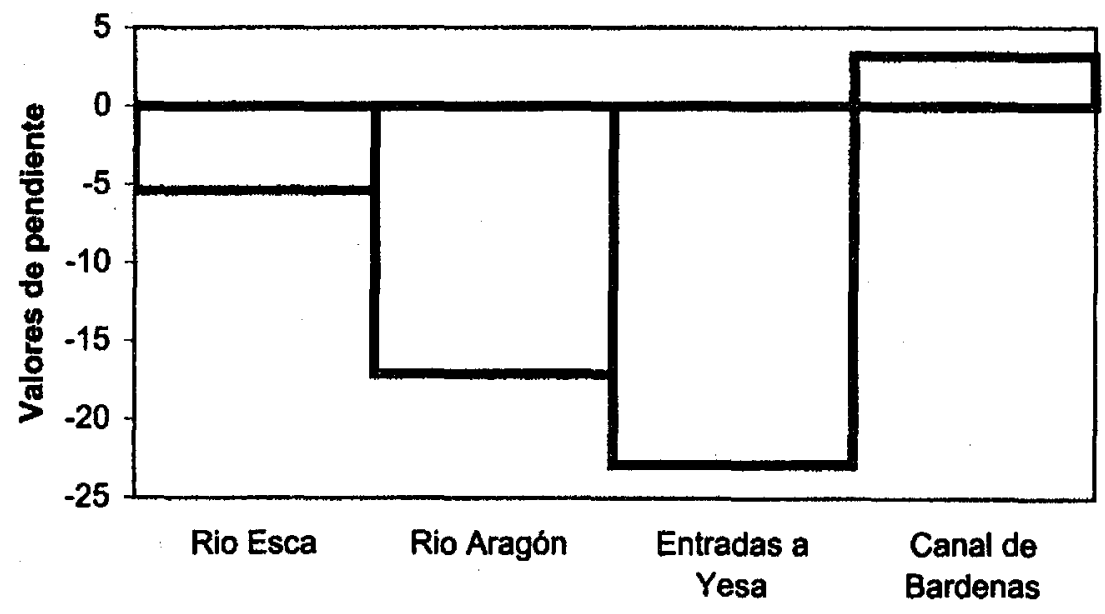

Figura. 6. Pendiente de las tendencias observadas en el caudal (1959-1996).

\section{Conclusiones}

El embalse de Yesa fue construido para atender las necesidades de abastecimiento de agua a los modernos perímetros de regadío de Bardenas, entre las provincias de Navarra y Zaragoza. Esa demanda exige que el embalse alcance el máximo volumen almacenado a finales de primavera (preferentemente junio), de manera que se pueda atender al riego estival. Ese volumen máximo se consigue reteniendo sobre todo caudales durante los periodos de aguas altas, que en la cuenca superior del río Aragón se centran sobre todo en primavera y, en menor medida, en otoño. Por lo tanto, el ritmo de llenado y vaciado anual del embalse de Yesa está 
representado por un patrón adaptado tanto a la demanda (necesidad de altos volúmenes embalsados antes del verano) como al régimen hidrológico. Además, aunque en teoría podría alcanzarse un mayor volumen embalsado durante el invierno, se prefiere mantener un margen de seguridad en previsión de posibles crecidas, y confiar en que el embalse acabará llenándose con las aguas de fusión y las lluvias primaverales. Esa previsión tiene, por otra parte, sentido en una cuenca en la que las influencias oceánicas, si no dominantes, sí tienen una importancia creciente hacia el sector occidental.

Sin embargo, aunque la demanda experimenta pocas fluctuaciones interanuales, el régimen fluvial presenta fuertes variaciones en función, sobre todo, de la mayor o menor intensidad de las lluvias (y avenidas) otoñales, de la acumulación-fusión de nieve y de las lluvias primaverales. En este trabajo se han identificado tres patrones diferentes de manejo del embalse de Yesa. El primero es el más adaptado aparentemente al régimen del río Aragón, con rápido incremento del volumen embalsado en otoño, suave incremento o estabilización en invierno y nuevo incremento en primavera. El segundo grupo, muy poco representado, implica escasos volúmenes embalsados en otoño, fuerte incremento a principios del invierno y nuevo aumento en primavera; se trata de un modelo en el que las lluvias de otoño son de muy baja intensidad y en cambio las invernales son más elevadas de lo normal. Finalmente el tercer grupo muestra un suave y continuo incremento desde octubre hasta junio, pero los volúmenes embalsados están por debajo de los otros grupos.

Una cuestión de gran interés es que los diferentes patrones de gestión han dominado en diferentes periodos de tiempo, siendo el primero característico de las décadas de los sesenta y setenta, mientras el tercero lo es de las décadas de los ochenta y noventa. Dado que la demanda no ha variado sustancialmente, es evidente que se ha producido un cambio relevante en el régimen de los ríos Aragón y Esca. La Fig. 6 ha demostrado que en efecto, la tendencia de las aportaciones es negativa, mientras la de las salidas por el canal de las Bardenas permanece prácticamente estable. La Fig. 5 señala que entre el grupo 1 y el grupo 3 la mayor diferencia en cuanto a las entradas de agua en el embalse estriba en la marcada reducción de los caudales otoñales. De igual forma, las entradas primaverales son notablemente inferiores en el grupo 3. En consecuencia, la única posibilidad de alcanzar una reserva de agua relativamente elevada consiste en retener caudales durante el invierno, lo que obliga a mantener bajos los caudales de salida hacia el río Aragón. Las razones de esta evolución, no identificadas en este trabajo, son de vital importancia para explicar no sólo las tendencias observadas, sino también para predecir la evolución futura de los recursos hídricos en ésta y en otras cuencas próximas.

\section{Agradecimientos}

Los datos de volumen almacenado en el embalse de Yesa y caudales de los aforos de la cuenca alta del río Aragon Cuenca de Yesa, han sido facilitados por la Confederación Hidrográfica del Ebro. Este trabajo ha sido elaborado con el apoyo de los siguientes proyectos de investigación: "Water resources managment in a changing environment: the impact of sediment on sustainability" (WARMICE-ENV4-CT98-0789), financiado por la Comisión Europea, 
"Estaciones permanentes para el estudio de procesos hidrológicos en ambientes mediterráneos"- EPROHIDRO (HID98-1056-C02-01) e "Identificación de fuentes de sedimento y áreas generadoras de escorrentía en relación con los cambios de uso del suelo"- HIDROESCALA (REN2000-1709-C04-01/GLO), financiados por la CICYT.

\section{Bibliografia}

AL-TAIEE, T.M., 1990. The influence of a dam on the downstream degradation of a river bed: case study of the Tigris River. In Hydrology in Mountainous Regions II-Artificial Reservoirs: Water and Slopes (R.O. Sinniger \& M. Monbaron, eds.), LAHS Publ.,194: 153-160.

ANDERS BRANDT, S., 2000. Classification of geomorphological effects downstream of dams. Catena, 40: 375-401.

CREUS, J., 1983. El clima del Alto Aragón occidental. Instituto de Estudios Pirenaicos, 233 pp., Jaca.

GARCÍA RUIZ, J.M. \& LASANTA T., 1993. Land-use conflicts as a result of land-use change in the Central Spanish Pyrenees. Mountain Research and Development, 13(3): 295-304.

GARCÍA RUIZ, J.M., PUIGDEFÁBREGAS, J. \& CREUS, J., 1985. Los recursos bídricos del Alto Aragón. Instituto de Estudios Altoaragoneses, 224 pp., Huesca.

GARCÍA RUIZ, J.M., PUIGDEFÁBREGAS \& CREUS, J., 1986. La acumulación de nieve en el Pirineo Central y su influencia hidrológica. Pirineos, 127: 27-72.

GIERS, A., FREISTÜHLER, E. \& SCHULTZ, G., 1998. Methodology for assesment of ecohydrological effects of dam construction in a headwater region. In Hydrology, Water resources and Ecology in Headwaters (K. Kovar, U. Tappeiner, N.E. Peters \& R.G. Craig, eds.), LAHS Publ., 248, 509-514.

JIONGXIN, X., 1990. Downstream hydrological effects of reservoirs built in mountainous areas and their environmental influences. In Hydrology in Mountainous Regions. II-Artificial Reservoirs: Water and Slopes (O. Sinniger \& M. Monbaron, eds.), LAHS Publ., 194: 187-194.

HARDEN, C., 1993. Land Use, soil erosion, and reservoir sedimentation in an andean drainage basin in Ecuador. Mountain Research and Development, 13(2):177-184.

HIGGS, G. \& PETTS, G., 1988. Hydrological changes and river regulation in the UK. Regulated Rivers: Rearch and Management, 2: 349-368.

MCINTYRE, S. C., 1993. Reservoir sedimentation rates linked to long-term changes in agricultural land use. Water Resources Bulletin, 29: 487-495.

MORRIS, G. \& FAN, J., 1997. Reservoir Sedimentation Handbook. Mc Graw Hill, 1065 pp., New York.

PETKOVIC, S., DRAGOVIC, N. \& MARKOVIC, S., 1999. Erosion and sedimentation problems in Serbia. Hydrological Sciences Journal, 44(1): 63-77.

PIRCHER, W., 1990. The contribution of hydropower reservoirs to flood control in the Austrian Alps. In Hydrology in Mountainous Regions. II- Artificial Reservoirs: Water and Slopes (R.O. Sinniger \& M. Monbaron, eds.), LAHS Publ., 194: 3-10. Lausanne.

SUMMER, W., 1990. Transportation and deposition of sediment in the reservoir of the Altenwoerth Hydropower plant on the Danube. In Hydrology in Mountainous Regions. IIArtificial Reservoirs: Water and Slopes (R.O. Sinniger \& M. Monbaron, eds.). LAHS Publ., 194:107-112. 
TAMBURINO, V., BARBAGALLO, S. \& VELLA, P., 1990. Evaluation of sediment deposition in Sicilian artificial reservoirs. In Hydrology in Mountainous Regions. II-Artificial Reservoirs:Water and Slopes (R.O. Sinniger \& M. Monbaron, eds.), LAHS Publ., 194: 113-58.

THOMAS, D. H. L., 1996. Dam construction and ecological change in the riparian forest of the Hadejia-Jama'are floodplain, Nigeria. Land Degradation and Development, 7: 279 295.

VALERO GARCÉS, B., NAVAS, A., MACHÍN, J. \& WALLING, D., 1998. Sediment sources and siltation in mountain reservoirs: a case study from the Central Spanish Pyrenees. Geomorpbology, 28: 23-41.

VERSTRAETEN, G. \& POESEN, J., 2000. Estimating trap efficiency of small reservoirs and ponds: methods and implications for the assessment of sediment yield. Progress in Pbysical Geography, 24(2): 219-251.

ZSUFFA, I., 1999. Impact of Austrian hydropower plants on the flood control safety of the Hungarian Danube reach. Hydrological Sciences Journal, 44(3): 363-371. 